\title{
High performance simulation of dynamic hydrologic exchange and implications for surrogate flow and reactive transport modeling in a large river corridor
}

\author{
Yilin Fang ${ }^{1}$, Xuehang Song ${ }^{1}$, Huiying Ren ${ }^{1}$, William A \\ Perkins $^{1}$, Pin Shuai ${ }^{1}$, Jason Hou ${ }^{1}$, Jie Bao ${ }^{1}$, Xingyuan \\ Chen $^{1}$, and Timothy D Scheibe ${ }^{1}$ \\ ${ }^{1}$ Pacific Northwest National Laboratory, Richland WA \\ ( ${ }^{*}$ Correspondence: yilin.fang@pnnl.gov)
}

Hydrologic exchange flows (HEFs) are important environmental processes in riverine ecosystems. Key factors that influence the spatial and temporal variations of HEFs include river stage, riverbed morphology, and riverbed hydraulic conductivity. However, their impacts have often been evaluated only independently or on small scales. In this study, we evaluate the combined interactions of these factors on HEFs using a high-performance subsurface flow and transport simulator (PFLOTRAN) for a river corridor of 51 $\mathrm{km}^{2}$ along a reach of the Columbia River in Washington, US. Spatially distributed hourly river stage data are applied to the riverbed boundary of the three-dimensional model. Riverbed sediments are classified into four facies, to which heterogeneous hydraulic conductivity can be assigned. Seven hydromorphic units were mapped to the riverbed based on field observations and two-dimensional river flow simulations. Subsurface residence times of HEFs were estimated by particle tracking using $100 \mathrm{M}$ particles injected at random riverbed locations and times. Transient storage models (TSMs) that are widely used to study streamsubsurface exchanges at reach scale can be considered simplified or surrogate versions of the high-resolution model. Our results show that that hyporheic exchange flow rates are significantly lower than the upwelling and downwelling exchange flow rates and there is potential to parameterize surrogate models such as TSMs to incorporate hydromorphicunit-scale process understanding to advance mechanistic understanding of river corridor processes at reach to watershed scales. Assuming constant concentration of stream tracer and a single hyporheic storage zone, the annual average of stream tracer mass within the hyporheic zone calculated using TSM is greater than $90 \%$ of that from the 3D simulation. However, the mass discrepancies between models become large for non-conservative solutes, which suggests that a multirate mass transfer model may be warranted to take into account the variable biogeochemical consumption rates along flow paths of different residence times. 